Citation: Serdar, H., "Clustering Research in Fifth Generation Mobile Communication Networks". Journal of Engineering Technology and Applied Sciences 5 (1) 2020 : 25-31.

\title{
CLUSTERING RESEARCH IN FIFTH GENERATION MOBILE COMMUNICATION NETWORKS
}

\author{
Hasan Serdar \\ Department of Computer Engineering, Faculty of Seydisehir Ahmet Cengiz Engineering, Necmettin \\ Erbakan University, Konya \\ hserdar@erbakan.edu.tr
}

\begin{abstract}
Clustering procedures are utilized to broaden the life of systems and increment vitality proficiency. In this study, some clustering algorithms and traffic expectations consider in 5G communication systems are inspected. There is a need to explain how to improve the nature of client experience through clustering. Understanding the requirements of clients is basic to give the capacity to help various situations in smart frameworks. Client mindfulness or client situated plan is a challenge in clustering. Inquires about have demonstrated that the usage of clustering plans in 5G systems presents difficulties and that clustering procedures created with insightful system determination arrangements can be of extraordinary advantage. The present investigations are not perfect in unique frameworks with a wide assortment of client situations since they are performed in both homogeneous and low-level heterogeneous systems and can't work. Also, when the 5G happens, the issue will turn out to be more mind-boggling than customarily rearranged. Different challenges identified with the execution of clustering methods in 5G networks are introduced and examined.
\end{abstract}

Keywords: 5G, clustering, wireless networks, communications

\section{Introduction}

The fifth generation wireless communication technology is improving the future pervasive wireless systems administration, applications, and user experiences. Ideal models are picking up energy in 5G technology details, as they bring cloud local applications closer to end users and system associated things, empowering new classes of administrations with challenging execution necessities [1,2]. To satisfy the consistently expanding need for information moving, every telecommunication organization, just as worldwide institutionalization elements, are effectively driving the innovative work of the fifth generation of wireless communications $[3,4,5]$. 
Clustering in telecommunication frameworks, showed up in the system of specially appointed networking, which was proposed as a promising answer for infrastructure denied networks, for example, crisis communication power outages or to convey fit for wireless networks $[3,6]$. A clustering calculation can parcel sensors into various clusters. In each cluster, a cluster header is chosen to be accountable for producing a transmission plan, gathering information from every one of the sensors in the cluster and transmitting the collected information back to the base station. Clustering procedures are likewise one of the ways to deal with topology control, which can sort out a network into a cluster based network. Various algorithm methods can be executed in this structure so as to accomplish explicit targets $[1,7,8,9]$.

This study offers a review walkthrough on clustering procedures and the most applicable research exertions during the earlier years. In this paper, additionally examined the difficulties of applying clustering procedures into Internet of Things dependent on 5G systems.

The rest of this paper is organized as follows: Fifth Generation Mobile Communication Networks is introduced in Section 2. Clustering Algorithms are given in Section 3. Results and Discussion is given in Section 4. Finally, the conclusions of this paper are drawn in Section 5.

\section{Fifth generation mobile communication networks}

The Fifth Generation wireless communication technology is proposed for future wireless networking, applications, and clients' nature of experience. So, 5G wireless communication need give higher network limit, accomplish associations with diminished postponement and cost, and vitality contrasted with existing wireless innovations. The current networks are experiencing an assortment of costly and exclusive hardware, just as hard state flagging conventions. At the point when a specific capacity isn't accessible, the cell administrator need to supplant existing hardware regardless of whether it is as yet adequate for most purposes, which demonstrates the trouble of altering the administration. Consequently, the 5G wireless communication framework is required to offer practically identical network limit, and simultaneously bolster associated gadgets with various mobile administrations.

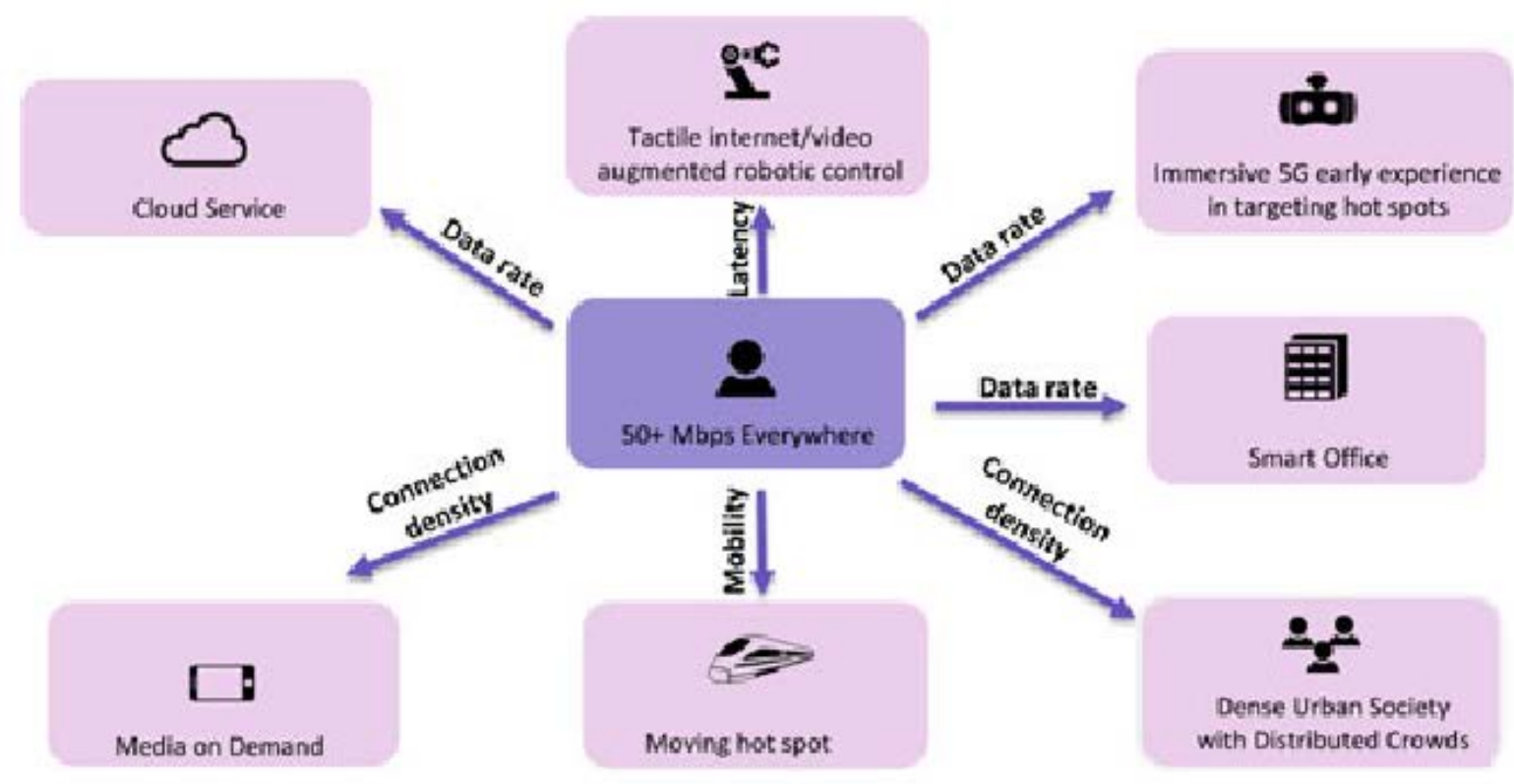

Figure 1. 5G network use cases [10] 
A few 5G use cases have been recognized in various administrative bodies and research programs where there are continuous endeavors to orchestrate them. The absolute most significant use cases that may profit by the millimeter wave advancements, on the entrance, backhaul, or both, are laid out in Figure 1 [10]. In the wide range various groups is reasonable for various use cases. Use cases served in lower portion of spectrum for tactile internet or automated control though higher of this range utilized for extraordinary mobile broadband family, cloud administration or 5G early experience. These use cases set stringent necessities on the networks decreased inactivity.

\section{Clustering algorithms}

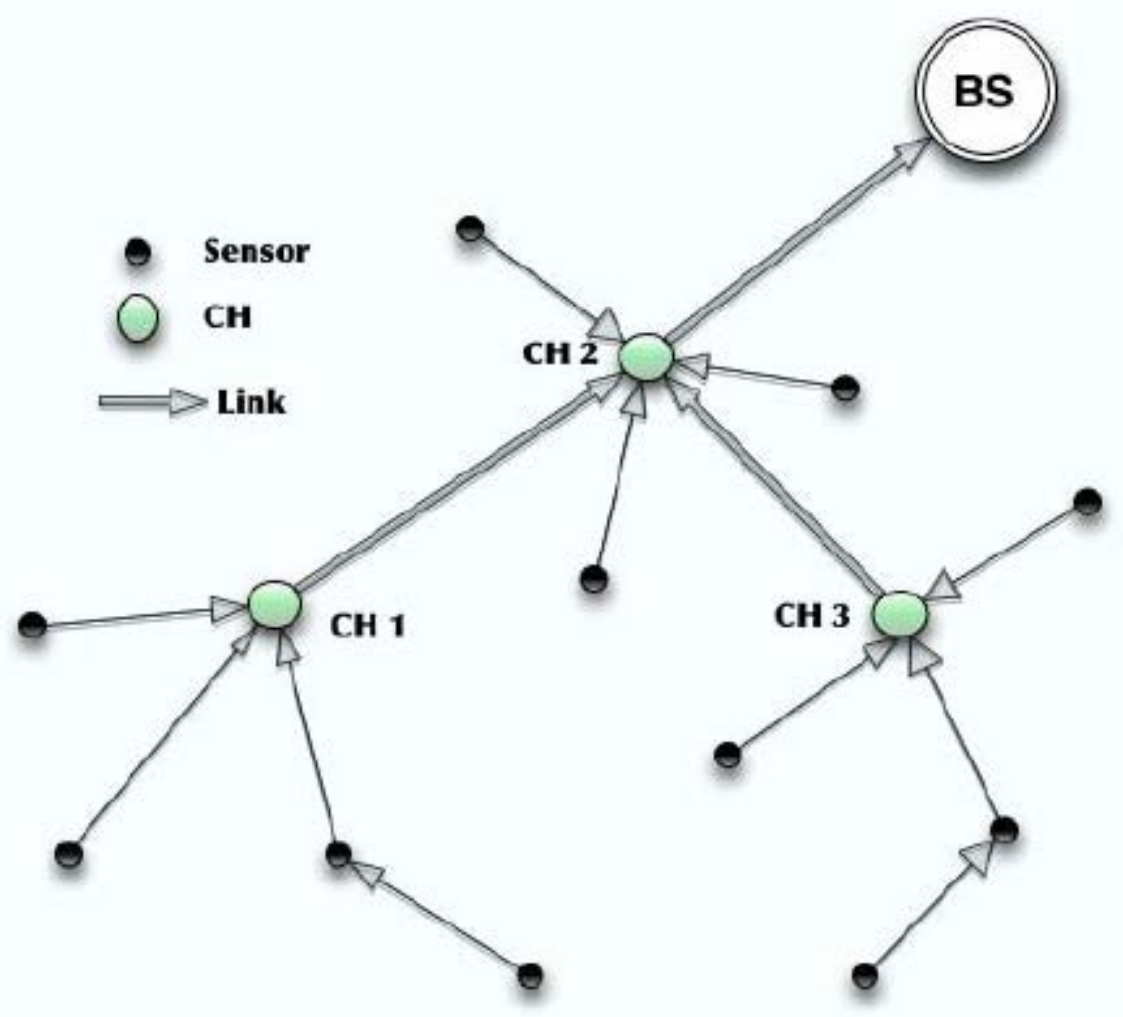

Figure 2. A cluster based network example [11]

Topology control should be connected to adjust the network load, increment the network versatility, and delay the network lifetime for wireless networks. Clustering strategies are deal with topology control, which can sort out wireless networks into a cluster based network. A clustering calculation can parcel sensors into various clusters, as appeared in Figure 2. In each cluster, a cluster header is chosen to be accountable for creating a transmission plan, gathering information from each sensors in the cluster and transmitting information back to the base station. In clustered structure, the framework can keep up a more drawn out life by booking the obligation cycle between the sensors inside a cluster.

The advantage of clustering is the stability of cooperative connections. Clusters are framed so as to amplify the time accessibility of clusters, thus expanding the accessibility of agreeable connections. An efficient arrangement of clusters takes a simpler organization of noninterfering channels. All nodes share a typical control channel for cluster arrangement in clustered networks and support while various channels are allotted for information 
transmission. Table 1 abridges the most significant advantages of clustering while at the same time featuring the expenses and structure contemplations of applying clustering procedures.

Table 1. Advantages of clustering algorithms [3]

\begin{tabular}{|c|c|}
\hline Benefits & \\
\hline Routing complexity & Sets a smaller backbone formed between clusterheads and gateways to reduce the number of possible routing paths \\
\hline Interference avoidance & $\begin{array}{l}\text { Favors non interfering channel allocation between adjacent clusters. Channel allocation algorithms are easily deployed in } \\
\text { clustered networks. }\end{array}$ \\
\hline Collision avoidance & $\begin{array}{l}\text { Contention-free communication strategies can be deployed for intra-cluster communication. Typically CDMA and TDMA are } \\
\text { suggested for communication between culster-members and the clusterhead. }\end{array}$ \\
\hline Topological information & $\begin{array}{l}\text { The routing information kept by a node in a cluster is limited to the configuration of that cluster. Inter-cluster routing } \\
\text { information can be managed by clusterheads and gateways. }\end{array}$ \\
\hline Relay selection & $\begin{array}{l}\text { Clusterheads in a cluster are assumed to be more stable than the rest of the nodes. They are therefore suitable to be relay } \\
\text { nodes. }\end{array}$ \\
\hline Coordination & $\begin{array}{l}\text { A clusterhead can favor coordination and synchronization for cooperative tasks (i.e. resource management or localization } \\
\text { mechanisms) }\end{array}$ \\
\hline \multicolumn{2}{|l|}{ Costs } \\
\hline Overhead & $\begin{array}{l}\text { Information to form and maintain the topology is periodically exchanged, increasing energy consumption. Periodicity of this } \\
\text { information is a trade-off between energy and stability. }\end{array}$ \\
\hline Complexity & Apart from the computation complexity, in some algorithms the number of rounds for completeness is not bounded. \\
\hline Stationary assumption & $\begin{array}{l}\text { Information exchanged by nodes to perform the clustering algorithm is supposed to not vary before the completion of the } \\
\text { algorithm. }\end{array}$ \\
\hline Ripple effect & Strict optimization of the clustering topology can lead to a frequent re-clustering process, thus decreasing stability \\
\hline \multicolumn{2}{|l|}{ Design considerations } \\
\hline Number of hops & $\begin{array}{l}\text { There is a trade-off between stability and communication cost that depends on the number of hops between cluster-members } \\
\text { and clusterheads. }\end{array}$ \\
\hline Cluster size & $\begin{array}{l}\text { There is a trade-off between inter-cluster and intra-cluster communication that depends on the number of clusters and the } \\
\text { number of members per cluster. }\end{array}$ \\
\hline Inter-cluster connectivity & $\begin{array}{l}\text { The number of gateways presents a trade-off involving energy, reliability and routing complexity. The optimum number of } \\
\text { gateways depends on the mobility and density of the network. }\end{array}$ \\
\hline
\end{tabular}

\section{Results and discussion}

The Fifth Generation wireless communication technology, and Clustering Algorithms have been presented previous sections. Now themes of this research will be presented in this section and the deficiency in the literature on this subject will be eliminate.

Table 2. Themes

\begin{tabular}{|l|c|l|}
\hline Themes & Studies, $\mathbf{n}(\%)$ & References \\
\hline $\mathbf{5 G}$ & $30,(94 \%)$ & $\begin{array}{l}{[1,2,3,4,5,6,7,8,9,10,11,12,13,14,15,16,17,18,19,21,23,24,25,26,} \\
27,28,29,30,31,32]\end{array}$ \\
\hline $\begin{array}{l}\text { 5G radio } \\
\text { access }\end{array}$ & $2,(6 \%)$ & {$[10,26]$} \\
\hline Channel & $23,(72 \%)$ & $\begin{array}{l}{[3,4,5,7,10,12,13,14,15,16,17,18,19,20,21,23,25,26,27,28,29,31} \\
32]\end{array}$ \\
\hline Clustering & $32,(100 \%)$ & $\begin{array}{l}{[1,2,3,4,5,6,7,8,9,10,11,12,13,14,15,16,17,18,19,20,21,22,23,24,} \\
25,26,27,28,29,30,31,32]\end{array}$ \\
\hline $\begin{array}{l}\text { Clustering } \\
\text { algorithm }\end{array}$ & $18,(56 \%)$ & {$[3,5,6,7,8,9,11,12,13,20,21,22,23,24,25,29,31,32]$} \\
\hline Networks & $29,(91 \%)$ & $\begin{array}{l}{[1,2,3,4,5,6,7,8,9,10,11,12,13,14,15,16,17,18,21,22,23,24,} \\
25,26,27,28,29,30,31]\end{array}$ \\
\hline $\begin{array}{l}\text { Wireless } \\
\text { network } \\
\text { virtualization }\end{array}$ & $2,(6 \%)$ & {$[15,16]$} \\
\hline
\end{tabular}


Clustering techniques are provide a platform for network topology management and better network. Some good algorithms was proposed in [11] (Balanced Energy-Efficiency clustering algorithm), in [12] (CAVDO and MA-DTR-based clustering techniques), in [13] (ELA-WF soft clustering algorithm), in [22] (LEACH DCHS CM algorithm), in [31] (Routing algorithm).

References that include only classification procedures do not propose new algorithms, but examine existing algorithms.

Further research was conducted to determine clustering algorithms in fifth generation mobile communication networks. Themes of this research summarized in Table 2. The present investigations are not perfect in unique frameworks with a wide assortment of client situations since they are performed in both homogeneous and low level heterogeneous systems and can't work [1-32].

Also, when the 5G happens, the issue will turn out to be more mind boggling than customarily rearranged. Inquires about have demonstrated that the usage of clustering plans in 5G systems presents difficulties and that clustering procedures created with insightful system determination arrangements can be of extraordinary advantage [1-32].

\section{Conclusions}

In this study, clustering algorithms in fifth generation mobile communication networks were investigated. Purpose of this work is to examine the clustering algorithms in fifth generation mobile communication networks in field of engineering articles and some papers. The articles and papers reviewed were classified into seven categories. These categories are; 5G, 5G radio access, channel, clustering, clustering algorithms, networks, wireless network virtualization. The results of the articles and papers show that usage of clustering plans in 5G systems presents difficulties and that clustering procedures created with insightful system determination arrangements can be of extraordinary advantage. In the articles reviewed, the fifth generation mobile communication networks were explained and the results of the current studies were shared.

\section{References}

[1] Bruschi, R. et al., "A multi-clustering approach to scale distributed tenant networks for mobile edge computing”, IEEE Journal on Selected Areas in Communications, 37(3) (2019) : 499-514.

[2] Le, L.-V. et al., "Applying big data, machine learning, and SDN/NFV to 5G traffic clustering, forecasting, and management” In 2018 4th IEEE Conference on Network Softwarization and Workshops (NetSoft) (2018) : 168-176.

[3] Sucasas, V. et al., “A survey on clustering techniques for cooperative wireless networks”. Ad Hoc Networks, 47 (2016) : 53-81.

[4] Chun, Y.J. et al., "A comprehensive analysis of 5G heterogeneous cellular systems operating over shadowed fading channels", IEEE Transactions on Wireless Communications, 16(11) (2017) : 6995-7010. 
[5] Ke, S. et al., “An adaptive clustering approach for small cell in ultra-dense networks”. In 2017 9th International Conference on Advanced Infocomm Technology (ICAIT) (2017) : 421-425.

[6] Alawe, I. et al., “An efficient and lightweight load forecasting for proactive scaling in 5G mobile networks”, In 2018 IEEE Conference on Standards for Communications and Networking (CSCN) (2018) 1-6.

[7] Paramonov, A. et al., "Clustering optimization for out-of-band d2d communications”, Wireless Communications and Mobile Computing, (2017).

[8] Ouyang, Y. et al., “APP-SON: Application characteristics-driven SON to optimize 4G/5G network performance and quality of experience”, In 2017 IEEE International Conference on Big Data (Big Data) (2017) : 1514-1523.

[9] Xu, L., O’Hare, G. \& Collier, R., “A smart and balanced energy-efficient multihop clustering algorithm (smart-beem) for mimo iot systems in future networks”, Sensors, 17(7) (2017) : 1574.

[10] Shariat, M. et al., "5G radio access above $6 \mathrm{GHz}$ ", Transactions on Emerging Telecommunications Technologies, 27(9) (2016) : 1160-1167.

[11] Xu, L., Collier, R. \& O’Hare, G.M., “A survey of clustering techniques in WSNs and consideration of the challenges of applying such to 5G IoT scenarios", IEEE Internet of Things Journal, 4(5) (2017) : 1229-1249.

[12] Aadil, F. et al., "Clustering algorithm for internet of vehicles (IoV) based on dragonfly optimizer (CAVDO)”, The Journal of Supercomputing, 74(9) (2018) : 4542-4567.

[13] Balevi, E. \& Gitlin, R.D., "A clustering algorithm that maximizes throughput in 5G heterogeneous F-RAN networks", In 2018 IEEE International Conference on Communications (ICC) (2018) : 1-6.

[14] Chun, Y.J., Cotton, S.L., Dhillon, H.S., Ghrayeb, A., et al., “A stochastic geometric analysis of device-to-device communications operating over generalized fading channels”, IEEE Transactions on Wireless Communications, 16(7) (2017) : 4151-4165.

[15] Feng, J. et al., “An approach to 5G wireless network virtualization: architecture and trial environment”, In 2017 IEEE Wireless Communications and Networking Conference (WCNC) (2017) : 1-6.

[16] Feng, Z. et al., “An effective approach to 5G: Wireless network virtualization”, IEEE Communications Magazine, 53(12) (2015) : 53-59.

[17] Ge, X. et al., "User mobility evaluation for 5G small cell networks based on individual mobility model”, IEEE Journal on Selected Areas in Communications, 34(3) ( 2016) : 528-541.

[18] Gharbieh, M. et al., "Self-organized scheduling request for uplink 5G networks: A D2D clustering approach”, IEEE Transactions on Communications, 67(2) (2018) : 1197-1209.

[19] Haneda, K. et al., "5G 3GPP-like channel models for outdoor urban microcellular and macrocellular environments”, In 2016 IEEE 83rd Vehicular Technology Conference (VTC Spring) (2016) : -7.

[20] He, R. et al., "An automatic clustering algorithm for multipath components based on Kernel-power-density”, In 2017 IEEE Wireless Communications and Networking Conference (WCNC) (2017) : 1-6. 
[21] Khan, Z. et al., “Two-level cluster based routing scheme for 5G V2X communication”, IEEE Access, 7 (2019) : 16194-16205.

[22] Liu, Y. et al., “A cluster maintenance algorithm based on LEACH-DCHS protoclol”, In 2008 International Conference on Networking, Architecture, and Storage (2008) : 165166.

[23] Maatouk, A. et al., "Graph theory based approach to users grouping and downlink scheduling in FDD massive MIMO”, In 2018 IEEE International Conference on Communications (ICC), (2018) : 1-7.

[24] Mathew, A.P., Arthi, M. \& Babu, K.V., "An uniform clustering based coverage and cost effective placement of serving nodes for 5G”, In 2017 International Conference on Innovations in Electrical, Electronics, Instrumentation and Media Technology (ICEEIMT), (2017) : 106-110.

[25] Moreno Garc'lia-Loygorri, J. et al., "Wideband channel modeling for mm-wave inside trains for 5G-related applications”, Wireless Communications and Mobile Computing, (2018).

[26] Pateromichelakis, E. et al., "Interference management enablers for $5 \mathrm{~g}$ radio access networks”, In 2016 IEEE Conference on Standards for Communications and Networking (CSCN), (2016) : 1-7.

[27] Ribeiro, F.C. et al., "Clustered multiuser detection for the uplink of 5G systems”, In 2016 IEEE 83rd Vehicular Technology Conference (VTC Spring) (2016) : 1-5.

[28] Saha, R.K., Nanba, S. \& Nishimura, K., "A technique for cloud based clustering and spatial resource reuse and scheduling of 3D in-building small cells using CoMP for high capacity CRAN”, IEEE Access, 6 (2018) : 71602-71621.

[29] Sangodoyin, S. et al., "Cluster characterization of 3-D MIMO propagation channel in an urban macrocellular environment”, IEEE Transactions on Wireless Communications, 17(8) (2018) : 5076-5091.

[30] Wang, X. et al., "Clustering of virtual network function instances oriented to compatibility in 5G network”, China Communications, 14 (12) (2017) : 111-119.

[31] Wang, Z., Qin, X. \& Liu, B., “An energy-efficient clustering routing algorithm for WSNassisted IoT”, In 2018 IEEE Wireless Communications and Networking Conference (WCNC) (2018) : 1-6.

[32] Zhang, P. et al., "Cluster-based analysis of wideband millimeter-wave channel for corridor environment”, In 2017 Sixth Asia-Pacific Conference on Antennas and Propagation (APCAP) (2017) : 1-3. 